this difficulty, it is found, can readily be overcome. All measurements with alternate currents relate to mean values. It is not necessary to make the instantaneous value of the torque exerted on the moving system of the instrument a measure of the simultaneous value of the quantity tested, as, for instance, is the case with ordinary dynamometers. All that is needed is proportionality between the mean values. With the aid of this principle it can be shown that the shunt magnet offers for instrument purposes great advantages, one of these being that the density of the air-gap flux, without loss of accuracy, can be increased to a value far exceeding that obtainable in permanent magnet instruments.

The ordinary modes of analysis used for the solution of alternate current problems rest upon several convenient but inaccurate assumptions, such as sine law wave form, proportionality of magnetic flux to magnetising current, \&c. Such methods can be used to give an approximate explanation of known results, but are not adapted to predict precisely the action of a new type of instrument in terms of measured data relating to its parts. In a paper read before the Royal Society ${ }^{2}$ on January 16 , a simple form of analysis, previously pointed out by the present writer, is further developed. This method is free from

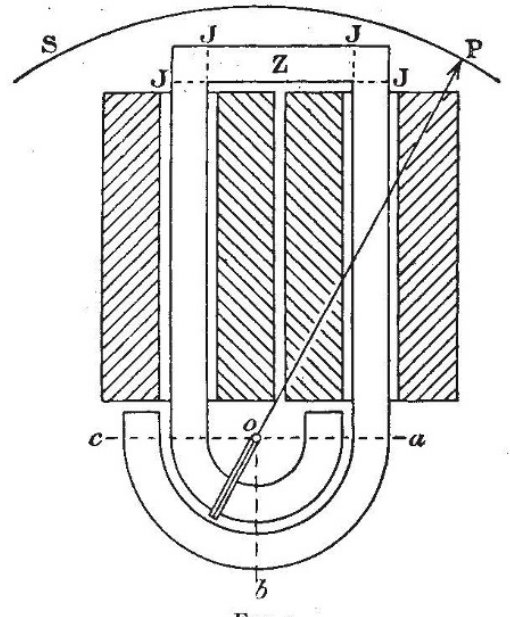

F1G. r.

assumptions such as those mentioned. It rests upon the theorem that each one of a number of known cyclic quantities, however different these may be in wave form, can be expressed as a linear function of an equal number of other cyclic quantities, the latter being such that the mean square of each is unity, and the mean product of any two is zero. This theorem leads naturally to a vector method of representation, which in many cases closely approximates to that usually adopted for alternate current investigations, but there is the important difference that when the results obtained are independent of assumptions such as sine law wave form this fact can be proved, while if such independence does not hold good a superior limit can be found to the error involved in results obtained by the ordinary method.

One of the instruments of which the behaviour has been investigated theoretically and fully confirmed experimentally is illustrated in Fig. $\mathrm{I}$. The magnetic circuit consists of a block of thin iron stampings shaped so as to have only one narrow air gap, the section of which is increased by. suitably. extending the poles. The field windings are shown in section, and are liberally supplied with copper. By this construction the ratio of resistance to impedance is made small. The moving coil is rectangular, one side turning in the air gap, the opposite side acting as an axis perpendicular to the figure, and shown at 0 . The instrument becomes a voltmeter if the moving coil is placed in series with a condenser across the voltage applied to the field coil. It becomes a wattmeter if the main current is passed through an induction coil the secondary of which

1 "Alternate Current Measurement." By Dr. W. E. Sumpner. (Communicated by Prof. Perry, F.R.S.)

NO. 2OI 3 , VOL. 78 ] contains a large resistance and the moving coil. If the resistance of the field coil were zero, the flux through the core would simply depend on the applied voltage, whatever the magnetic properties of the core; and if this coil were short-circuited the core flux could not be varied. The chief points to be investigated were the effect of the actual resistance of the field coil, the influence of the E.M.F. induced by the field in the moving coil circuit, and the precise meaning and influence of the self-inductance of the moving coil. Exact formulæ have been established and verified for all these effects, some of which may be illustrated by the following tests. If the field coil be opencircuited and a current be passed through the moving coil, this will turn so as to enclose the greatest flux, but this position will not be oc because of the portion of the flux crossing the air gap twice, this part being greatest for the position $o b$. For feeble currents the resulting position will be near $o b$, but will tend to approach oc for larger currents, owing to the corresponding increase in the permeability of the core. If, however, the field coil be short-circuited, an alternating current through the moving coil will invariably turn it to the position $o b$, because such current cannot produce a flux through the long limbs of the magnet. If the field coil be excited by an alternating voltage and the moving coil circuit be closed through a small resistance, this coil, owing to its self-inductance, will turn to $o a$ so as to enclose the minimum flux, while if under the same conditions the moving coil circuit be closed through a condenser, the coil will turn to $o c$ so as to enclose the maximum flux.

A thorough analysis of these effects, confirmed by actual tests, shows that the instrument can be so constructed that for most purposes its errors are negligibly small, and also that it is possible to eliminate precisely these errors for any specified frequency by using a special winding round the magnet with its ends joined up to a condenser the capacity of which is iefermined by the frequency and the winding. None of the ext:- arise from the variable magnetic properties of the core. In connection with alternating current work, the voltage-controlled magnetic field thus offers great advantages for investigating purposes.

W. E. SUMpner.

\section{NEW LIGHTS ON THE ANTHROPOLOGY OF CALIFORNIA.}

THE anthropological department of the University of California, thanks to Mrs. Hearst's munificent endowment, is able to issue a further series of studies on the native races of that State. The most voluminous contribution is that of Mr. S. A. Barrett on the "Ethnogeography of the Pomo and Neighbouring Indians," a group of tribes numbering at present about 1000 souls, and occupying the region known to geographers as the Coast Range Mountains immediately north of San Francisco Bay, and running eastward to the Sacramento River. These people are now partially civilised, and support themselves by farming and labour. But sufficient is known of their primitive condition to show that they had no totemic clans or groups, and that their tribal organisation was weak, there being no chief in the commonly accepted sense of the term. There was a sort of council of minor chiefs presided over by a chief captain, whose authority was strictly limited, and who was elected by the community. The inferior chiefs, on the other hand, held their offices by hereditary right, and the succession passed from one incumbent to the family of that sister who was nearest to him in age, kinship and descent being in the female line.

In regard to culture, these people fall into three divisions:- the ocean tribes, who depended for food upon the fish and molluses which abounded in the sea, and derived the material for their food and clothing from the redwood forests of the coastal districts; secondly, the valley tribes, who occupied in severe weather round grassthatched houses, while during the summer they wandered along the streams and lived in temporary brush shelters, but used no canoes, as the rivers are of inconsiderable volume: lastly, the tribes of the inner lake region, who built elliptical huts thatched with the tule rush, which 
also supplied them with materials for nets, mats, and slings with which they killed water-fowl, using for the purpose of fishing canoe-shaped rafts of the tule. The materials for the study of this civilisation now extinct are found in the ancient village sites which have been carefully examined by Mr. Barrett. The greater part of his elaborate report is occupied by a survey of the tribal dialects, which adds largely to the information recorded by Schoolcraft, Powell, Bancroft, and others. In another and less elaborate report Messrs. S. A. Barrett and A. L. Kroeber give an account of the Miwok tribe.

Of more general interest and importance is the investigation conducted by Mr. W. J. Sinclair into the question of the existence of relics of prehistoric man in the auriferous gravels of the Sierra Nevada. The evidence of the early existence of man in California has been hitherto almost entirely based on the well-known report issued in 1880 by Prof. J. D. Whitney in the course of the geological survey of the State. The present inquiry has been devoted to a further investigation of the sites from which the human remains and objects alleged to be the work of man are said to have been derived. The result is seriously disconcerting to those who have relied on the evidence collected by Whitney. Mr. Sinclair points out that, though these gravels and the intercalated volcanic outflows are admittedly of various geological periods, Whitney made no attempt to indicate from which series of gravels the relics were obtained. In the case of the discoveries made in mines worked by hydraulic machinery the provenance of the vast majority of objects can no longer be verified, and there is good reason to suspect that many, if not all, the specimens have been washed down from modern Indian village sites situated on bluffs overhanging the pits, which were disintegrated by the powerful water currents. Mr. Sinclair reviews in detail a11 the more remarkable "finds" in this region. One large mortar is said to have been found in association with " a small oval tablet of dark coloured slate with a melon and leaf carved in bas-relief." But it exhibits no signs of wear from gravel friction; the scratches are all recent defacements, and the carving is said to show very evident traces of a steel knife-blade.

Special attention is naturally given to the Calaveras skull immortalised by Bret Harte, which is now a cherished possession of the Harvard Museum. Mr. Sinclair asserts that the substance adhering to it is not a gravel, but a cave breccia, and that the skull was not obtained in the gravels beneath the rhyolite, or from any other gravel of the rhyolitic epoch, none of which exhibits any trace of the stalagmitic cementation which has been recognised in the skull matrix. He suggests that the skull was derived from a comparatively modern Indian cave interment. If, he adds, man of a fairly high developed type was in existence during the deposition of these gravels, he must have been " a contemporary of the three-toed horse and other primitive forms of the late Miocene and early Pliocene, a thesis to which all geological and biological evidence is opposed." His conclusion is that the evidence is insufficient to prove the presence of the remains of men in the auriferous gravels; that there have been abundant opportunities for such relics to be accidentally mixed with these gravels; and that the local geological conditions render it improbable that such implements and bones have been found in the assumed sites.

It would be premature to attempt to criticise this important report in detail. Doubtless those authorities who assert the genuineness of these relics will not allow the case to go by default. English anthropologists have always adopted an attitude of reserve in relation to the Calaveras skull. If the question of the antiquity of the human race depended on the authenticity of these Californian discoveries, the position would perhaps be serious. But even if Mr. Sinclair's indictment survives the criticism to which it is inevitable that it must be exposed, the abundant evidence from other unquestioned sources which now exists renders a challenge of one set of relics a matter of little importance. Whatever may be the result of the controversy, the necessity of caution in dealing with evidence which has been collected without rigid scientific supervision is sufficiently obvious.
METEORIC PHENOMENA IN JUNE.

IN June there are few meteors seen which leave streaks. Possibly the twilight is responsible for this; it must partially be so, but $I$ do not think that it will wholly account for the seeming rarity of meteors of the same type as the August Perseids or November Leonids.

Yet there are a few noticed by vigilant observers, and especially in the morning hours. These are directed from radiants in Pisces, Andromeda, Aries, Perseus, and other constellations in the same general region of the sky. There are the $\alpha-\beta$ Perseids, $\delta$ Cassiopeids, $\pi, \mu$, and $\gamma$ Andromedids, $\beta$ Triangulids, $\alpha$ Arietids, $\beta$ and $\epsilon$ Piscids, and many others. In 1887 I saw several fine streak-leaving meteors from a radiant near the position of the August Perseids at about $\eta$ Persei. All the centres referred to, however, stand in need of corroboration, as they are supported on very slender evidence indeed.

Fireballs are not uncommon to June, though few real paths appear to have been hitherto determined in this month.

When we have such excellent atmospheric conditions as prevailed in June, 1887 , it is possible to discern a large number of showers. Some of these are directed from places south of the equator; for instance, there are radiants at $25^{\circ}-21^{\circ}, 269^{\circ}-24^{\circ}, 283^{\circ}-13^{\circ}, 305^{\circ}-12^{\circ}$, \&c.

Occasionally there are some late Virginids and Serpentids noticed in June, and a sprinkling of Ursa Majorids will be recorded by the careful observer.

The weather usually prevailing is excellent, and the sky, though light, is attractive. The writer has often been struck with the prominent aspect of the Milky Way on midsummer nights. It stretches nearly overhead, and the rich regions in Cassiopeia, Cepheus, and Cygnus are often beautifully displayed at this season of the year, notwithstanding the lack of suitable darkness.

\section{W. F. Denning.}

\section{VISION AND COLOUR-VISION.}

THE difference in physiological effect between incident and reflected light is a commonplace to those who have investigated the influence of surroundings on sensitive animals. The influence $d u$ fond is a function of the altered light reflected from these surroundings upon the eyes or skin of animals capable of assuming variable coloration. The direct incident light has little or no effect in producing the result.

In a series of papers (Zeit. f. Augenheilkunde, Bd. xvi. pp. 448-463; Wiener medezinische Wochenschrift, No. 46, 1906, No. 48, 1907) Prof. Raehlmann asserts that the light perceived by vertebrates is reflected from the plane separating the outer laminated limb of the rods and cones into the inner homogeneous limb of the same. He now claims that colour-vision is due to the reflected light setting up "stationary waves" in the inner limb of these retinal elements. To support this view he proceeds to show from recent work that if a Lippmann gelatin film (placed between a mirror and a source of light) be exposed to different parts of the spectrum, and then cut into microscopic sections and examined under a high power, the substance of the film is seen to be traversed by zones of black granules separated by colourless intervals, the interspaces equalling the wave-length of the light used, and, therefore, whilst perceptible in the case of red and yellow light, are so crowded together in green and blue light as to be almost continuous. This result is claimed by Prof. Raehlmann as confirmatory evidence of the truth of his hypothesis. The sensitive film is represented by the morphologically inner portion of the rod or cone, the mirror by the membrane separating this from the outer refractive portion, and the case would be complete if any arrangement of black and colourless zones comparable to that set up in the film could be discovered in the inner portion of the cone, but though Prof. Raehlmann has used the ultra-microscope after a given monochromatic exposure, he can discover nothing in the inner cone-limb but a perfectly uniform granulation, and though the length of the cone is known to vary with that of the light-waves used, yet he has not been able to correlate the somewhat 